\title{
Beitrag zur Kenntnis der Keratomycosis aspergillina.
}

\author{
Fon \\ Dr. Martin Zade, \\ Assistent der Klinik. \\ Nit Taf. XVIII, Fig. $1-3$.
}

Das klinische Bild der Keratomycosis aspergillina bietet, zum mindesten in einem gewissen Stadium der Entwicklung, gewisse Merkmale dar, welche im Hinblick auf die bisher bekannt gewordenen Fälle am Menschen und auf die besonders durch Leber $(1,2)$ eingehend untersuchten Veränderungen am Kaninchenauge wohl zur Wahrscheinlichkeitsdiagnose schon bei oberflächlicher Betrachtung führen können. Aber selbst wenn wir annehmen wollen, dass die klinischen Merkmale für den, der dies seltene Bild gesehen hat, wohl charakterisiert sind, so ist doch im Wesen der Erkrankung noch manches der Aufklärung bedürftig.

Während die zuerst beschriebenen Fälle als durchaus schwere Affektionen bezeichnet wurden, haben sich später mehrere leicht verlaufende Erkrankungen nachweisen lassen. Während es sich in den meisten Fällen um Aspergillus fumigatus gehandelt hat, werden in einigen Fällen andere Pilze genannt. Widersprechend sind auch die Angaben über die Therapie der Schimmelpilzkeratitis. Bedenkt man dabei, dass die Zahl der bisher bekannt gewordenen Fälle nur etwa 22 ist, von denen nicht alle einer genauen Untersuchung unterzogen worden sind, so ergibt sich von selbst die Forderung, jeden neuen Fall besonders zu beachten.

Der Fall, der in folgendem mitgeteilt werden soll, bot in mancher Hinsicht Besonderheiten.

Fran Anna R., 44 Jahre alt, ländliche Arbeiterin, kam am 8. XI. 1906 mit einer Hypopyonkeratitis des linken Auges in die Poliklinik. Sie gab 
an, dass ihr am 3.XI.1906 beim Dreschen etwas ins linke Auge geflogen sei, wahrscheinlich eine "Hafer-Spelze". Ob etwas im Auge geblieben war, war ihr nicht bekannt. Am nächsten Abend stellten sich Schmerzen ein. Früher hat sie immer gut gesehen.

Es bestand heftige conjunctivale und pericorneale Injektion, dazu etwas Chemosis conjunctivae bulbi. An der Hornhaut zeigte sich temporal, etwa $1 \mathrm{~mm}$ vom Limbus entfernt, ein Geschwür von etwas ovaler Form, etwa $3 \mathrm{~mm}$ im senkrechten, $2 \mathrm{~mm}$ im herizontalen Durchmesser. Rand anffallend scharf, nicht erhöht. Der Geschwürsgrund war mit einer grauweissen Masse angefüllt, die leicht prominent war und bei Lupenbetrachtung krümlig und trocken aussah. Ein Fremdkörper war nicht sichtbar. Nasal vom Uleus bestand eine dasselbe wie eine Sichel umgebende tiefe Infiltration, zwischen der und dem Geschwür eine $1 / 2 \mathrm{~mm}$ breite Zone klar und durchsichtig war. Im übrigen war die Hornhant normal. Hypopyon dickflüssig, 1,5 mm hoch. Iris heftig entzündet, Pupille mit Atropin nur mittelweit, Synechien nicht sichtbar. Tränensack und Conjunctiva ohne Besonderheiten.

Das rechte Auge bot bei guter Sehschärfe normalen Befund.

Das Conjunctivalsekret enthielt nur einige Stäbchen der Diphtheriegruppe. Auch auf Kulturen vom Conjunctivalsekret wachsen aur XeroseBacillen. In einigen mit Fremdkörpernadel vom Geschwürsgrund entnommenen Partikeln zeigten sich keine Bakterien, nur dichtes Geflecht feiner Faden liess sich nachweisen. Am besten liessen sich diese Fäden im ungefärbten Präparat (Einschluss in $10 \%$ Glycerinlösung) betrachten. Zunächst konnte man wohl erkennen, dass ein Pilzmycel vorlag, da aber zuerst keine Fruktifikationsorgane gefunden wurden, konnte die genaue Diagnose nur durch die Kultur erbracht werden. Es wurden von den dem Geschwürsgrund entnommenen Teilchen einige auf je ein Agar- und ein Blutserumröhrchen gebracht. Schon nach 24 Stunden war bei $37^{\circ}$ eine Entwicklung von Aspergillus fumigatus vor sich gegangen. Herr Dr. Miehe, Privatdozent am botanischen Institut, dem ich auch an dieser Stelle verbindlichst danke, konnte die Diagnose bestätigen.

Der Krankheitsverlauf war sehx hartnäckig. Versuche, die Geschwürsmasse mechanisch zu entfernen, wie es in mehreren der veröffentlichten Fälle gelungen ist ${ }^{1}$ ), brachten nur einzelne zähe, schwer zerzupfbare Bröckel zutage. Besonders am Rand war die Pilzmasse mit dem Hornhantgewebe eng verbunden. Behandlung mit Wasserstoffsuperoxyd, mit Aufstreuen von Europhenbor brachte keine Besserung.

Patientin wurde deshalb am 11. XI. 06 in die Klinik aufgenommen.

Das Geschwür nahm ganz allmählich an Grösse zu; zugleich bildet sich um den Infiltrationsring (den typischen Leberschen Einwanderungsring) ein zweiter ebensoleher aus, der den ersten konzentrisch, $1 \mathrm{~mm}$ von ihm entfernt, umgab und tief im Hornhautgewebe sass (am deutlichsten am 22. XI.). Es wurde nun kauterisiert. Am 25. XI. nochmalige Kauterisation. Es trat jetzt anch oberflächliche Hornhanttrïbung im Umkreise des Pilzrasens auf, die aber jetzt noch den zweiten tiefen Infiltrationsring er-

1) Es war deshalb wohl berechtigt, den Geschwürsgrund abzukratzen, ohne eine Schädigung fürchten zu müssen. 
kennen liess. In den nächsten Tagen weiterer Progress; fast $2 / 3$ der Hornhaut waren vom Pilzrasen eingenommen, der Rest war homogen dicht getrübt und liess jetzt keine Einzelheiten mehr erkennen. Eine Demarkationsrinne bildete sich nicht aus.

Es wurde nun (4. XII.) die Spaltung nach Saemisch vorgenommen und das Hypopyon herausgelassen. Es enthielt mikroskopisch nur Fibrin und Leukocyten.

Jetzt trat langsam Rüekgang der entzündlichen Erscheinungen ein. Es erfolgte allmählich Narbenbildung mit Hinterlassung einer vorderen Synechie.

29. XII. 06. S. L. Handbew. in $1 \mathrm{~m}$, Projektion prompt.

3. I. 07. Entlassung mit Xeroformsalbe. Kein Reizzusand mehr, Leukom von ${ }^{2} / s$ Hornhantgrösse.

4. II. 07. Wiederaufuahme. S. L. Handbew. in $2 \mathrm{~m}$, Projektion prompt.

5. II. 07. Iridektomie nach oben anssen.

9. II. 07. S. L. $=$ Fingerzahlen in $2 \frac{1}{2} \mathrm{~m}$.

Während dieses Krankheitsverlaufs wurden wiederholt Teile vom Geschwürsgrund abgekratzt und teils auf Nährböden verteilt, teils frisch in Glycerin untersucht. Auf den Nährböden (Glycerinagar und Serum) wuchsen ausnahmslos Reinkulturen von Aspergillus fumigatus. Von den mit Glycerineinschluss untersuchten Präparaten zeigten zwei die typischen Fruktifikationsorgane des Aspergillus fumigatus.

Man sah in den ungefärbten Präparaten ein dichtes Geflecht von Mycelien; an den Randteilen, wo die Pilzfäden weniger dicht waren, lagen vielfach weisse Blutkörperchen dazwischen.

Die Präparate, welche Fruktifikationsorgane enthielten, waren dem Zentrum des Pilzrasens entnommen. Während die den Randpartien entnommenen Pilzteile keine dieser Bildungen aufwiesen, waren sie hier ăusserst zahlreich (Taf. XVIII, Fig. 1). Der typische Bau der Konidienträger mit den die Endblase bedeckenden unverzweigten distal strebenden Sterigmen war unverkennbar ${ }^{1}$ ). Vielfach waren die abgeschnürten Konidien zum Teil in Reihen an den Sterigmen sichtbar. Das Mycel, das die Zwischenräume ausfüllte, zeigte hin und wieder Endanschwellungen. Dazwischen lagen massenhaft abgefallene Konidien. Da das Zentrum des Pilzrasens der Hornhaut nicht in toto, sondern nur in einzelnen Partikeln abgehoben wurde, liess sich allein aus diesem Befund nichts genaues über die Lage der Fruktifikationsorgane aussagen. Doch ergibt sich aus verschiedenen, später zu erörternden Gründen, dass dieselben ganz obertächlich sassen.

Besonders um die Frage nach dem Vorkommen der Frukti-

1) Durchschnittliche Dicke des Konidienträgers am Stiel $5 \mu$, an der Endblase (ohne Sterigmen) $10 \mu$. 
fikationsorgane, das nach dem bisher in der Literatur Bekannten abgelehnt werden musste ${ }^{1}$, zu entscheiden und auch um die Pathogenität nachzuweisen, wurden Tierversuche vorgenommen. Am Kaninchenauge wurde nach Bildung einer Tasche in der Hornhaut mit der Lanze das infektiöse Material in diese Tasche hineingebracht.

Während hierzu ausschliesslich Reinkulturen von Aspergillus fumigatus, der aus der Hornhaut der Patientin gezüchtet war, verwandt wurden, wurde an einem Kaninchenauge in die durch Lanzenschnitt gebildete Tasche ein durch Abkratzung rom Ulcus der Patientin gewonnenes Partikelchen eingebracht. Das andere Auge war tags zuvor mit Kultur geimpft. Es zeigte sich nun, dass das mit auf künstlichem Nährboden gezüchteten Pilzmassen infizierte Auge viel geringfügiger erkrankte, als das andere durch Übertragung vom kranken Auge der Patientin infizierte. Während ersteres nach drei Tagen erst eine Pilzrasenbildung erkennen liess, war hier schon am nächsten Tage ein grosser Pilzrasen entwickelt; am zweiten Tage nach der Impfung stellte sich hier schon der Infiltrationsring ein, der dort erst am vierten Tage auftrat. Auch kam es im letzten Falle neben Hypopyonbildung zu heftiger Glaskörpereiterung, die im ersten fehlte, in dem sich nur ein kleines Hypopyon entwickelte. Der anatomische Befund beider am gleichen Táge enucleierten Augen zeigte noch deutlicher die Heftigkeit der entzïndlichen Erscheinungen auf dem mit Ulcusabschabungsmaterial geimpften Ange. Hier war die Pilzentwicklung in der Hornhautsubstanz viel umfangreicher und tiefer vor sich gegangen.

Die Beurteilung dieser Erscheinung muss allerdings mit einiger Vorsicht geschehen. Denn man hat es nicht in der Hand, ein messbares Quantum keimfähigen Materials einzuimpfen, und selbst wenn das einigermassen möglich wäre, liesse sich nicht erwarten, dass alles an Ort und Stelle haften bleibt. Auch ist es wohl nicht gleichgültig, von welchem Teil der Kultur entnommen wird, ob mehr Sporen oder mehr Mycel eingeimpft wird. Dass spezifische Giftwirkungen dabei eine Rolle spielen (etwa analog der bei vielen Mikroorganismem durch Tierpassage zu erreichenden Virulenzsteigerung), ist nicht ausgeschlossen, nachdem erst kürzlich Otto (26) bei zwei aus Italien stammenden Schimmelpilzstämmen

1) In der während der Drucklegung dieser Arbeit erschienenen Bakteriologie des Auges von Axenfeld (27) wird ebenfalls angegeben, dass Fruktifikationsorgane am Auge nicht sicher beobachtet sind. 
Giftstoffe gefunden hat; fünf Stämme, die aus Deutschland stammten, gaben kein positives Resultat. Jedenfalls soll dieser Befund hier genannt werden, weil der Unterschied ein zu auffälliger war, als dass zufällige Momente zur Erklärung ausreichten.

Bei den Hornhautimpfungen am Kaninchen erhielt ich die typischen Pilzrasen, wie sie zuerst von Leber (2) beschrieben wurden. Von besonderem Interesse war mir die Frage, ob nicht auch beim Kaninchen Fruktifikationsorgane auf der Hornhaut gebildet würden, wie es in dem vorliegenden Krankheitsfall zweifellos geschehen war. Dabei konnte ich feststellen, dass von im ganzen 8 erfolgreichen Hornhautimpfungen bei zweien sich typische Konidienträger in dem Pilzrasen fanden. Wie unter anderem Fuchs (5) in seiner Arbeit hervorhebt, lässt der Pilzrasen in einem vorgerückten Stadium einen peripheren und einen zentralen Teil unterscheiden. Dieser letztere löst sich meist leicht von seiner Unterlage ab. Es gelingt nun beim Tier gewöhnlich leicht, ein solch zentrales Stück loszulösen (es hatte in meinen beiden Fällen eine Grösse von ungefähr $3 \mathrm{~mm}$ ), um es in $10 \%$ Glycerinlösung auf einem Objektträger auszubreiten. Das erste Mal wurde ein Deckglas mit sanfter Gewalt aufgedrückt, so dass die Masse breit gedrückt wurde. Mikroskopisch sah man ein sehr dichtes Pilzgeflecht mit vielen Sporen und obenauf vereinzelte Konidienträger, welche offenbar durch den Deckglasdruck deformiert waren. An der Form der zusammengepressten Endblasen liess sich erkennen, dass die Fruchtträger senkrecht zur Fläche des Pilzrasens aus diesem herausgewachsen waren (Tafel XVIII, Fig. 2). Es stellte nämlich die Endblase einen Kreis dar, der rings herum von Sterigmen umgeben war, ein Bild, das bei den Konidienträgern des Aspergillus fumigatus nur auf diese Weise zu stande kommen kann. Durch die Blase sah man auch den Stiel derselben hindurch ${ }^{1}$ ). In diesem Falle waren sieben Tage seit der Impfung vergangen, am vierten Tage war ein Einwanderungsring vorhanden gewesen, der zur Zeit der Ablösung des zentralen Sequesters nicht mehr ausgesprochen war.

In dem zweiten Fall, in dem sich die Konidienträger fanden, lagen ganz ähnliche Verhältnisse vor. Auch hier wurde ein zentraler Sequester in derselben Weise in Glycerin eingeschlossen; es wurde aber das Deckgläschen nur sanft aufgelegt. Mikroskopisch war die

1) Dass es sich hier um einen andern Fruchtträger als den des Aspergillus fumigatus gehandelt hat, ist ausgeschlossen, weil in jedem Falle nach Bildung des Ulcus durch Abimpfung und Übertragung auf Nährboden die Identität festgestellt wurde. 
Hauptmasse des Sequesters zu dick, um im durchfallenden Lichte Einzelheiten exkennen zu lassen, jedoch waren an der Peripherie mehrere typische, diesmal von der Seite zu betrachtende Konidienträger des Aspergillus fumigatus sichtbar. Auch hier sassen die Konidienträger ganz an der Oberfläche (Tafel XVIII, Fig. 3). Sie lagen auf einem, hier an der Peripherie nicht besonders dichten Mycelgeflecht, zwischen dem sehr viele Sporen lagen. Von den Sterigmen waren die meisten Sporen abgefallen. Das Mycel zeigte vielfache Endanschwellungen.

Es ist somit durch diese Untersuchungen festgestellt, dass auf der menschlichen Hornhaut wie auf der des Kaninchens Fruktifikationsorgane gebildet werden. Nach meinen Informationen ist das bisher nur von Ball (16) angegeben worden, ohne dass man ihm besonders Glauben schenkte (Kayser 17). Wenn man allerdings die Angaben Balls näher ins Auge fasst, so muss man zugeben, dass an seinem Falle viel Unklares ist. Er spricht von einem "Ulcus" und beschreibt dann ein anatomisches Bild desselben mit über den Pilzmassen wegziehendem Epithel (!). Unter dem Epithel, also von der Luft abgeschlossen, bildet er die Konidenträger ab. Da es aber feststeht, dass die Fruktifikationsorgane nur an der Luft gedeihen, und da Ball keine Kulturen angelegt hat, so muss wirklich an diesem Fall manches zweifelhaft bleiben.

Die Gründe, warum die Fruktifikationsorgane bisher in den zum Teil mit denkbar grösster Sorgfalt ausgeführten Untersuchungen der Autoren nicht gefunden wurden (siehe besonders Kayser Seite 56), sind meines Erachtens folgende:

1. Die Bildung der Konidienträger findet nur in einem vorgerückten Stadium der Pilzentwicklung statt, also etwa vom sechsten Tage ab. Sie ist ein Ausdruck von guten Wachstumsbedingungen; nur ein kräftig wucherndes Mycel ist im stande zu fruktifizieren. Nach den bisher negativen Resultaten konnte Fuchs mit Recht sagen, "endlich wäre hervorzuheben, dass die Schimmelpilze weder in der menschlichen noch in der Kaninchenhornhaut es zu Fruktifikationen bringen, woraus man auch auf schlechte Ernährungsund Wachstumsverhältnisse schliessen darfts. Also nur da, wo die Pilze gutes Fortkommen finden, werden wir erwarten dürfen, Fruktifikationen zu finden.

2. Die Bildung der Konidienträger geht nur im zentralen Teile des Pilzrasens und nur an dessen Oberfläche vor sich. Das ist von vornherein zu erwarten, wenn man die Schimmelpilzbildung auf der 
Hornhaut mit einer Kolonie auf künstlichem Nährboden vergleicht. In der Tat ist ja kein prinzipieller Unterschied vorhanden: ebenso wie auf dem Nährboden als tiefste Schicht der ihm aufliegenden Pilzmasse das dichte Geflecht des Thallus zu erkennen ist; diesem liegt dann die Masse der sogenannten Luftmycelien auf, aus denen, im Zentrum einer Kolonie, senkrecht zur Fläche des Nährbodens, die Fruchtträger hervorwachsen [Siebenmann (25)], genau ebenso geht das Wachstum der Konidienträger auf der Hornhaut auch nur in den oberflächlichsten zentralen Teilen vor sich.

3. Dass die Fruchtträgerbildung überhaupt, abgesehen von dieser Beschränkung auf eine bestimmte Stelle des Ulcus, noch durch andere Momente behindert ist, ist wahrscheinlich. Vielleicht vermag der Lidschlag eine mechanische Fortschwemmung herbeizuführen, vielleicht kommt auch der Tränenflüssigkeit eine hemmende Wirkung zu.

4. Es ist mir bisher nur in frischen, ung efärbten Präparaten gelungen, Konidienträger zu finden; die meisten Autoren aber, welche mikroskopisch untersucht haben, haben nach Fixierung und Härtung eingebettete Präparate gefärbt. Allerdings ist auch von manchen [Leber (1), Uhthoff und Axenfeld (7 und 8), Fuchs (5), Osterroht (22)] frisch untersucht worden, jedoch scheint das Hauptgewicht auf gefärbte Präparate gelegt zu sein. Der in Fig. 3 abgebildete Sequester wurde später in Paraffin eingebettet und geschnitten, es fanden sich in den Präparaten nur Mycelien, die Fruktifikationsorgane waren zerstört.

Wenden wir uns nun einigen andern bedeutungsvollen Punkten in der Kenntnis der Keratomycosis aspergillina zu.

Der Entstehungsmodus ist wohl kein strittiger Punkt mehr. Wenn auch nicht in allen Fällen eine Verletzung angegeben wurde, so muss sie doch als Bedingung angenommen werden [Osterroht (22) u. A.]; in einigen Fällen wurde sogar ein Fremdkörper gefunden [Uhthoff und Axenfeld (7), Kayser (17)]. Auch dass meist pflanzliche Stoffe die Träger des Infektionsstoffes sind, muss als Regel gelten. Ebenso steht es fest, dass die Pilzelemente an einer von Epithel entblössten Stelle der Hornhaut deponiert werden müssen, um zur Entwicklung zu gelangen.

Dass noch andere Erreger neben dem Pilz bei der Erkrankung eine Rolle spielen können, lässt sich a priori nicht von der Hand weisen, ist jedoch in ausgesprochenen Fällen höchst unwahrscheinlich [Leber (1) Seite 299]. Soweit Angaben darüber vorhanden sind, fanden sich bei Osterroht in einem von 5 Agarröhrchen Kulturen 
von Staphylococcus albus, in dreien wuchs Aspergillus fumigatus, ein Röhrchen blieb steril. Ebenso geben Collomb (11) (Xerose), Uhthoff und Axenfeld (8) (Xerose), Kayser (17) (Gramnegativer Diplococcus) und Martin (21) (Xerose) als Nebenbefund Bakterien an. In meinem Falle waren nur vom Conjunctivalsekret, nicht aber vom Geschwürsgrund Xerose-Bacillen zu erhalten.

Das klinische Bild ist, wie auch durch meinen Fall bestätigt wird, charakterisiert durch die scharfe Begrenzung des Ulcus, durch den mit trockenen krümligen Massen bedeckten, Geschwürsgrund und durch den Infiltrationsring. Rinnenbildung und Sequestrierung, die in meinem Falle fehlten, sind ebenfalls oft gefunden. Beteiligung der Iris und Hypopyonbildung sind, wie die leichteren Fälle von Uhth off und Axenfeld, Kayser, Johnson und Martin lehren, kein ständiges Sympton.

Der Verlauf ist in den meisten Fällen ein schwerer und langwieriger, doch geht es nicht an, diesen Verlauf als den gewöhnlichen und den erst einige Male (siehe oben) beobachteten leichteren Verlauf als Ausnahme hinzustellen. Dazu gehört ein grösseres kasuistisches Material $^{1}$ ). Es wäre wohl denkbar, dass in manchen Fällen die Hornhaut mit den in das Gewebe eingebrachten Pilzen fertig wird, noch ehe das Geschwür eine Grösse erreicht hat, bei welcher eine schnelle Heilung nicht mehr zu erwarten ist. Begünstigend zu solcher Eliminierung wirkt dabei vielleicht ergiebige Vaskularisation, die in einigen Fällen [Kayser (17), Johnson (20), Martin (21)] als Gefässbändchen beschrieben ist. Hat aber der Pilzrasen einmal einen bestimmten Grad der Entwicklung erreicht, so ist der schwere, torpide Verlauf nicht mehr hintanzuhalten.

Soviel aber scheint festzustehen, dass es auch in den hartnäckigsten Fällen kaum zu so schweren destruktiven Veränderungen kommt, wie z.B. nach ungünstig verlaufenden Pneumokokkengeschwüren. Allerdings teilt Markow (13) Ausgang in Phthisis bulbi mit, Buchanan (19) hat zur Enucleation schreiten müssen (hier wurde aber die Pilzart nicht bestimmt). Schirmer (9) hat ebenfalls enucleiert, jedoch handelte es sich um ein sonst schwer erkranktes Auge.

Die Sequestrierung scheint nicht immer einzutreten. Fälle, in denen sie ausgesprochen vorhanden war, sind die von Osterroht

1) Rollet und Aurand (24) schreiben in ihrer Arbeit über experimentelle Untersuchungen mit Schimmelpilzen, dass die Keratom. asp. ste ts als HypopyonKeratitis mit sehr langsamem, etwa 2-3 Monate sich hinziehenden Verlauf auftrete; sie erwähnen aber nicht die Fälle von Kayser und Johnson. 
(22), Martin(21), Kayser(17), Gentilini(12), Johnson(20), Uhthoff(4), Uhthoff und Axenfeld (7,8); nicht ganz leicht löste sich der Sequester bei Fuchs (5), Wicherkiewicz(15). In meinem Falle war eine Loslösung überhaupt nicht möglich.

Ob die Lage des Ulcus hiermit oder überhaupt mit dem klinischen Verlauf in besonderer Beziehung steht, ist nicht ohne weiteres klar. Osterroht meint, dass bei zentraler Lage eine schwerere Form auftrete, als bei peripherer, was durch meinen Fall allerdings nicht bestätigt wird, denn das Geschwür war vom Rande her fortgeschritten. Bei Lebers (1) Fall erstreckt sich das Ulcus "von der Mitte nach dem lateralen Rand". Bei Uhthoff (4) "präpupillär etwas excentrisch nach oben", bei Fuchs (5) ziemlich zentral (etwa $7 \mathrm{~mm}$ gross), bei Uhthoff und Axenfeld (7 u. 8) im ersten Fall ,subzentral", im zweiten Fall 2-3 $\mathrm{mm}$ vom Limbus entfernt (dies war der leicht verlaufende Fall), bei Schirmer (9) ,innen unten vom Zentrum der Cornea", bei Markow (13), Basso (14) und Wicherkiewicz (15) zentral, bei Kayser(17) unten aussen, bei Johnson(20) $1 \mathrm{~mm}$ vom inneren Cornealrand (2 leichte Fälle), bei Martin (21) „temporal unten nahe dem Limbus" (leichter Fall), bei Osterroht (22) zentral. Auch zur Entscheidung dieser Frage ist offenbar die Zahl der bekannten Fälle nicht hinreichend.

Wohl der wundeste Punkt in der Frage der Keratomycosis ist die Therapie. Siebenmann (25), der eingehende Untersuchungen über Otomycosen gemacht hat, empfiehlt für diese Salicylspiritus. Dass die Salicylsäure, die hierbei doch wohl das Heilmittel darstellt, in dieser Form am Auge anwendbar ist, kann wohl a priori als unwahrscheinlich gelten. In den bisher beobachteten Fällen ist wohl jeweils die von dem betreffenden Beobachter sonst bei Hornhautulcus geübte Therapie angewandt worden. Die Durchschneidung nach Saemisch, die auch im vorliegenden Falle vorgenommen wurde, dürfte in verzweifelten Fällen jedenfalls angezeigt sein, wennschon man sie möglichst vermeiden wird, da meist eine Deformierung der Hornhautwölbung, oft auch Synechien nicht ausbleiben.

Es soll zum Schlusse noch einmal hervorgehoben werden, dass nicht alle als Keratomycosis aspergillina aufgeführten Fälle genau genug untersucht und beschrieben sind, um als einwandsfrei hierher gehörig betrachtet zu werden. Es sollen deshalb alle mir bekannt gewordenen Fälle mit Berücksichtigung dieses Punktes aufgezählt werden.

1. Leber (1): Kulturen (zuerst Asp. glaue. angenommen, später als Asp. fumig. beschrieben). 
2. Uhth off-Berliner $(3,4)$ : Keine Kulturversuche, mikroskopisch Pilzmycelien.

3. Fuchs (5): Kultur.

4. Mauthner (6): Keine Kultur, Wahrscheinlichkeitsdiagnose auf Grund des klinischen Bildes.

5. Uhth off und Axenfeld (1896) (7): Kultur.

6. Schirmer (9): Keine Kultar, anatomisch Pilamycel.

7. Uhthoff und Axenfeld (1897) (8): Kultur.

8. Churchman (10): Original unzugänglich. Sporen.

9. Collomb (11): Kultur, im Deckglaspräparat Pilzfäden mit einzelnen

10. Gentilini (12), erster Fall: Kultur.

11. - zweiter Fall: Pilzâden mikroskopisch, keine Kultur.

12. - , dritter Fall: Kultur.

13. Markow (13): Kultur.

14. Basso (14): Kultur.

15. Wicherkiewiez (15): Kultur, angeblich Penicillium glancum (von Kayser angezweifelt). Epithel.

16. Ball (16): Keine Kultur, angeblich Fruktifikationen unter dem

17. Kayser (17): Kultur.

18. Ellet (18): Keine Kultur, angeblich Asp. niger.

19. Buchanan (19): Keine Kultur, Mycelium "höchstwahrscheinlich" von einer Aspergillusart.

20. Johnson (20): Kultur.

21. Martin (21): Kultur.

22. Osterroht (22): Kultur.

[23. Köllner (23): Keine Kultur.]

Als ganz sichere Fälle von Keratomycosis aspergillina, d. h. solche, bei denen der Kulturnachweis erbracht ist, sind danach zu betrachten die unter Nr. 1, 3, 5, 7, 9, 10, 12, 13, 14, 17, 20, 21, 22 aufgeführten, das sind einschliesslich des hier beschriebenen $14 \mathrm{Fälle}$, von den übrigen dürfte bei den meisten ebenfalls Aspergillus fumigatus vorhanden gewesen sein, abgesehen von einigen ganz unsicheren Fällen, wie z. B. der von Ball.

Meinem hochverehrten Chef, Herrn Geheimrat Sattler, spreche ich für sein förderndes Interesse meinen verbindlichsten Dank aus.

\section{Literaturverzeichnis.}

1) Leber, Keratomycosis aspergillina als Ursache von Hypopyonkeratitis. Arch. f. Ophth. 1879. Bd. XXV, 2 .

2) -, Die Entstehung der Entzündung, Leipzig 1891.

3) Berliner, Diss. 1882.

4) Uhth off, Partielle Nekrose der mensehlichen Hornhaut durch Einwanderung von Schimmelpilzen. v. Graefe's Arch. f. Ophth. 1883. Bd. XXIX, 3. 
5) Fuchs, Keratom. asperg. Wiener klin. Wochenschrift 1894. Nr. 17.

6) Nauthner, Gesellsch. d. Ärzte in Wien, Sitzung vom 26. I. 1894. Ref. Wien. klin. Wochensehr. 1894. Nr. 17.

7) Uhthoff und Axenfeld, Beiträge zur pathol. Anatomie und Bakteriologie der eitrigen Keratitis. Arch. f. Ophth. 1896. Bd. XLII.

8) Dieselben, Weitere Beiträge usw., ebenda. Bd. XLIV, 1.

9) Schirmer, Ein Fall von Schimmelpilzkeratitis. Arch. f. Ophth. 1896. Bd. XLII, 1.

10) Churchman, Aspergillar Keratitis. Intern. med. Magaz. Philadelphia.

11) Collomb, Un cas rare d'infection cornéenne, Thèse de Lyon 1901.

12) Gentilini, Drei neue Fälle von Keratitis aspergill. Beitr. zur Augenheilk., Heft 45.

13) Markow, Westnik oftalmol. 1900.

14) Basso, La Cheratomicosi per Asp. fumig. Annali di Ottalm. 1900.

15) Wicherkiewicz, Über eine Schimmelpilzerkrankung der Hornhaut. Arch. f. Augenheilk. 1900. Bd. XL.

16) Ba11, Ein Beitrag zur Keratom. asperg. Die ophth. Klinik 1901. Nr. 18.

17) Kayser, Beitrag zur Kenntnis der Keratom. asperg. Klin. Monatsbl. f. Augenheilk. 1913, 1, Bd. XLI.

18) Ellet, Aspergillar Kerat, The ophthalm. Record 1903.

19) Buchanan, Leslie, Ophth. Soc. of the Unit. Kingd. Sitzung 3. Juli 1903. Klin. Monatsbl. f. Augenheilk. 1903, 2. Bd. XLI.

20) Johnson, Ein weiterer Beitrag zur leichten Form der Keratom. asperg. Klin. Monatsbl. f. Augenheilk. 1903, 2. Bd. XLI.

21) Martin, Ein neuer Fall von Keratom. asperg. Arch. f. Augenheilk. 1904. Bd. L.

22) Oste rrohi, Beitrag zur Kasuistik der Keratom asperg. Berl. klin. Wochenschr. 1905. Nr. 7.

23) Kollner, Schimmelpilzerkrankung der Sklera. Zeitschr. f. Augenheilk. 1906. Bd. XVI, Heft 5.

24) Rollet et Aurand, Nouvelies recherches sur les Kératites asperg. Révue génér. d'Opht. 1905. Tome XXIV. Nr. 12.

25) Siebenmann, F., Die Fadenpilze und ihre Beziehungen zur Otomycosis. Diss. Wiesbaden 1883.

26) Otte, Giftwirkung einiger Stämme von Asp. fumig. u. Penic. glauc. usw. Zeitschr. f. klin. Mediz. 1906. Bd. LIX, 2-4.

27) Axenfeld, Die Bakteriologie in der Augenheilkunde. Jena 1907.

\section{Erklärung der Abbildungen auf Taf. XVIII, Fig. 1-3.}

Fig. 1. Konidienträger des Asperg. fumig. aus menschlicher Hornhaut.

Fig. 2 und 3 . Konidienträger aus Kaninchenhornhaut, in Fig. 2 ist derselbe von oben her zusammengepresst, der Stiel ist unter der Endblase sichtbar. In Fig. 3 sind zwei Konidienträger an Rande eines Sequesters sichtbar. Daneben Mycel mit Endansehwellungen und Sporen. 


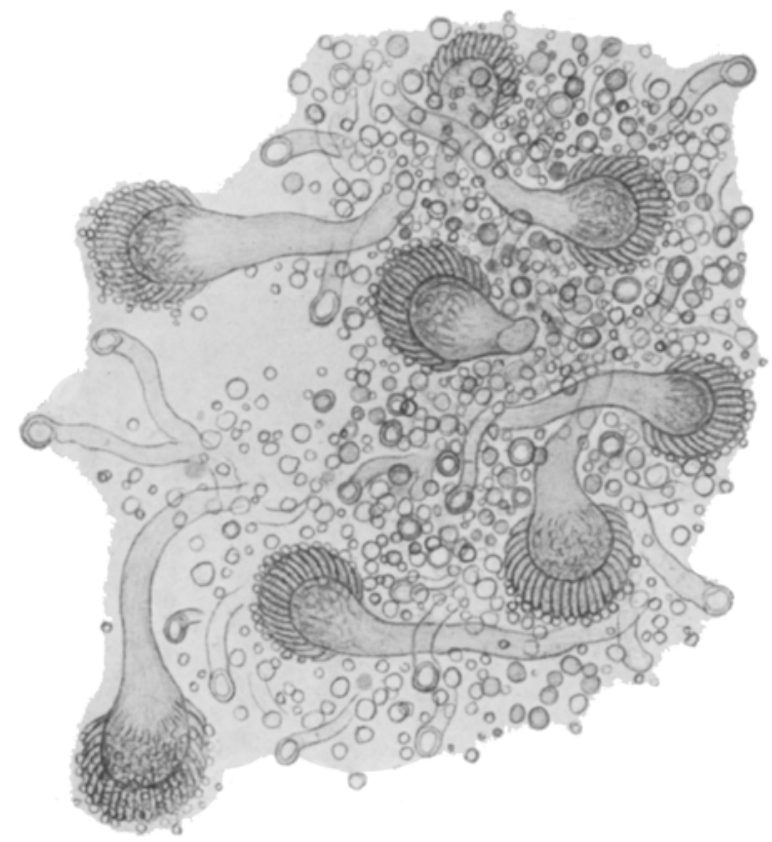

Fig. 1.

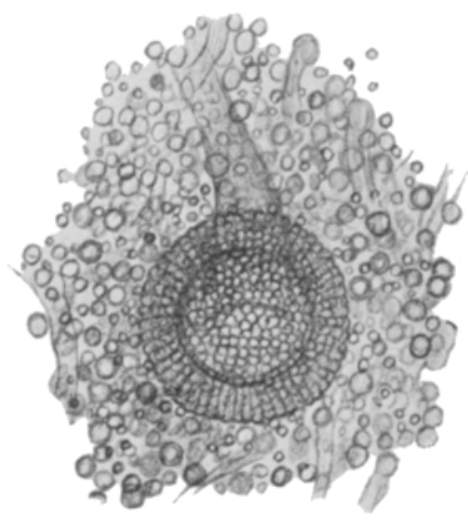

Fig. 2 ,

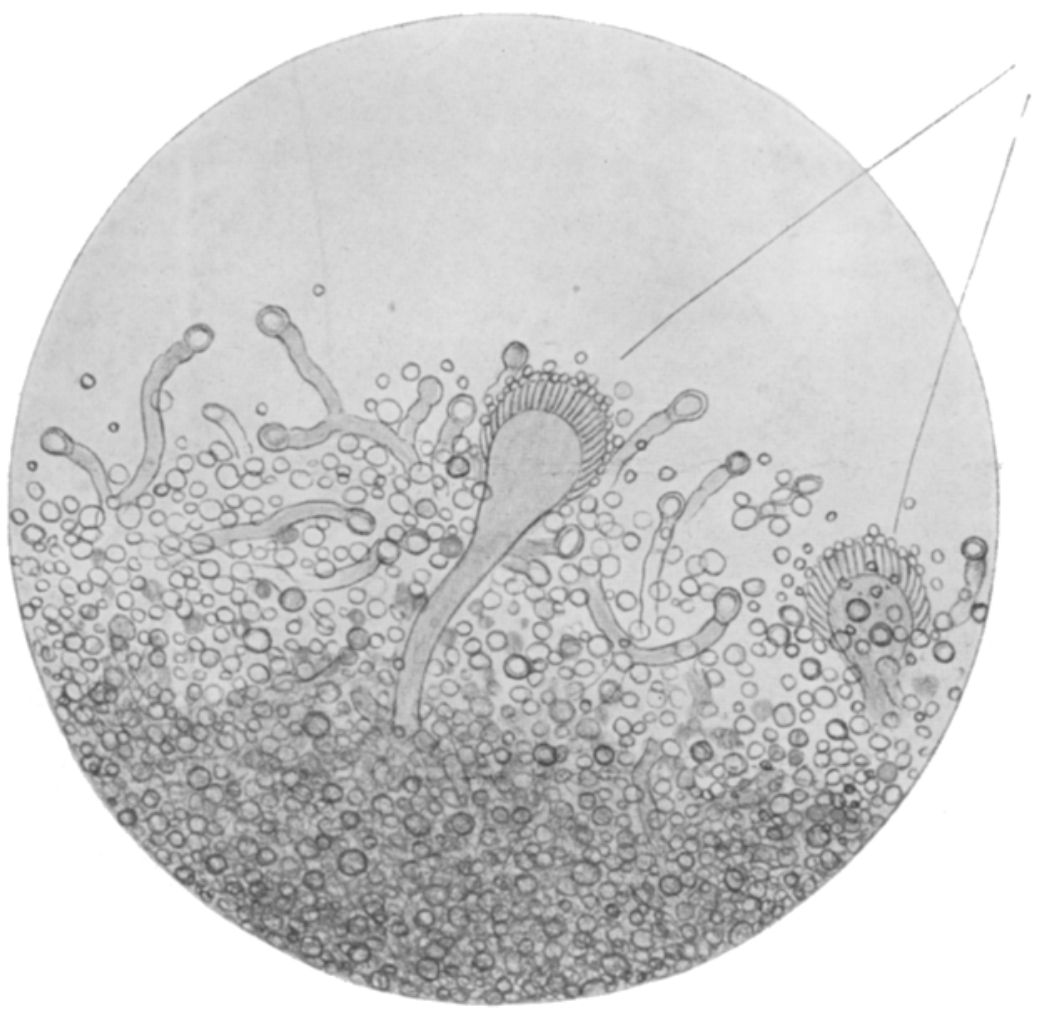

Fig. 3.

Verlag von Wilhelm Engelmann in Leipzig. 